\title{
Medicinal plants as source of antibacterial agents to counter K lebsiela pneumoniae
}

\author{
Gowsiya Shaik ${ }^{1}$, Sujatha $\mathrm{N}^{2}$ and Santosh Kumar Mehar ${ }^{1 *}$ \\ ${ }^{1}$ Department of Botany, Sri Venkateswara University, Tirupati-517502, Andhra Pradesh, India, ${ }^{2}$ Department of Microbiology, Sri Venkateswara University, \\ Tirupati-517502, Andhra Pradesh, India.
}

\section{ARTICLE INFO}

Article history:

Received on: 12/11/2013

Revised on: 10/12/2013

Accepted on: 27/12/2013

Available online: 30/01/2014

\section{Key words:}

Klebsiella pneumonia, antimicrobial activity,

Essential oil yielding plants.

\begin{abstract}
Several antibiotics are in use to treat different diseases caused by human pathogenic microorganisms. It is being observed that the invented medicines are not much effective in treatment, as the infection causing microbes are becoming resistant and modifying themselves into multidrug resistant strains. To fight against these human pathogens there is a need of introduction of new antibiotics. The synthetic derivatives or synthetic antibiotics are effective but unsafe for use and the results are not satisfactory due the resistance building capacity of the microbes. At the same time, the side effects and adverse reactions caused by these multispectrum antibiotics are not ignorable. Due to these reasons plant derived compounds or phytomedicines having antimicrobial action are needed to be evaluated, introduced and implemented through clinical and biological trials. Here we present the information about some plants which are reported to be antimicrobial in nature and act against human pathogens. The efficacy of their antimicrobial action needs to be evaluated for their biological functions. To be concise, our review is limited to the plants showing antimicrobial efficacy against Klebsiella pnuemoniae. For the present work we have focused on essential oil yielding plants which are known to be more useful to fight against this pathogen. A total of 90 plants species are reviewed, among them 14 are essential oil yielding plants. The plants which are able to inhibit the growth of the $K$. pneumoniae are enlisted and their details of the study such as family it belongs, parts used, microbial strain number used in the study, extractant and the standard antibiotic used to compare the effect are tabulated in detail to have a complete analysis of the information from the recent work. The essential yielding plants are tabulated separately.
\end{abstract}

\section{INTRODUCTION}

We are witnesses to an era where globalization and advancement in science is going on side by side with population increase. Infectious diseases are one of the leading causes of morbidity and mortality worldwide, especially in developing countries (Zeigler, 2005; Yala et al., 2001). Bacteria, in general, possess the genetic ability to acquire and transmit resistance to therapeutic agents. Following the massive use of antibiotics in human therapy, bacteria have developed several resistance mechanisms including the efflux of antibiotics (Yala et al., 2001). Several mechanisms have been proposed, such as target site modification, expression of the efflux pumps, and metabolic inactivation, which contribute to the drug resistance in MDR bacteria (Hooper, 2001).

\footnotetext{
* Corresponding Author

Santosh Kumar Mehar, Sri Venkateswara University, Tirupati-517502, Andhra Pradesh, India. Email: santoshkumar.1@rediffmail.com
}

According to World health organization (WHO) more than $80 \%$ of the world population relies on traditional medicine for their primary health care needs (Vashist and Jindal, 2012).

Not today, but from the ancient days itself, our ancestors depended on the plants for cure and medication. They have used several plants and their parts as treatment for different diseases. Plant based natural products traditionally known to combat microbial infections are expected to play a big role in this regard (Cowan, 1999).

Flavonoids isolated from natural dietary sources were also investigated in combination with antibiotics as a strategy against ESBL (Extended-Spectrum Beta-lactamase) producing clinical isolates of Klebsiella pneumoniae (Lin et al., 2005). Oregano essential oil, given in combination with fluoroquinolones, was found to enhance the activity of the drugs against ESBL-producing Escherichia coli (Si et al., 2008). 
Similar enhancement of antimicrobial activity in combination with plant derived terpenoids has been reported by Shahverdi et al. (2004) and Alimirzaee et al. (2009).

\section{A BRIEF INTRODUCTION ON Klebsiella pneumoniae Biology of K. pneumoniae}

Klebsiella pneumoniae is widely distributed in nature, found abundantly in soil and water. Klebsiella pneumonia is a Proteobacterian, included in Enterobacteriaceae. It is a Gramnegative bacterium, Cylindrical in shape and rod like. It is of about 2 microns in length and 0.5 microns in diameter (Fig 1). Its cells have a thick coat of slime or extracellular polysaccharide which is called a "capsule". The thickness of the capsule is approximately $160 \mathrm{~nm}$ in $K$. pneumoniae. The capsule protects the cells from desiccation, and may also protect them from phagocytosis when they are in an animal host. The structural genomics information of $K$. рпиетоniae can be read from the bioinformatics website http://kp.life.nthu.edu.tw/. K. pneumoniae has three subspecies with homologous DNAs but different biochemical reactions: $K$. pneumoniae subsp pneumoniae, K. pneumoniae subsp ozaenae, and $K$. pneumoniae subsp rhinoscleromatis. The characteristic biochemical reactions of these are:

$>K$. pneumoniae is lactose fermenting, $\mathrm{H}_{2} \mathrm{~S}$ - and indole negative, has a positive Voges Proskauer (VP) reaction, is capable of growth in $\mathrm{KCN}$ and uses citrate as a sole carbon source, and is incapable of growth at $10^{\circ} \mathrm{C}$.

$>$ The other two subspecies (ozaenae and rhinoscleromatis) are indole negative with a negative VP reaction.

$>K$ oxytoca, which is one of the other species of the genus, is indole positive, has a positive VP reaction, and is able to grow at $10^{\circ} \mathrm{C}$

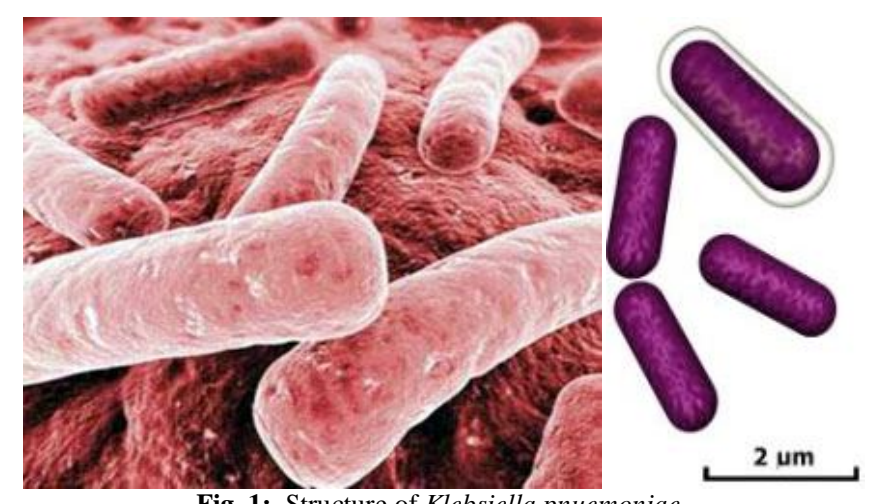

Fig. 1: Structure of Klebsiella pnиemoniae.

\section{Pathology of K. pneumoniae}

It was recognized over 100 years ago as a cause of community acquired pneumonia and is the opportunistic pathogen that can cause pneumonia, urinary tract infections, and bacteremia (Wu et al., 2012). Although the incidence of community acquired pneumonia caused by K. pneumoniae decreased for few years, but in humans with some immune related and other health problems such as diabetes mellitus, there is a health risk as the $K$. pneumoniae can cause an acute primary infection that result in lobar pneumonia. It is a common health care associated infectious agent which causes several infections, of which, the urinary tract, bloodstream, pneumonia and intra abdominal infections have become enormously common. Community acquired primary pyogenic liver abscess (PLA) caused by $K$. pneumonia is an emerging disease receiving increasing attention since past 20 years. Patients with this infection can present with or without septic metastatic complications (Keynan and Rubinstein, 2007). It is also involved in surgical site infections, peritonitis, pyogenic liver abscess, thoracic empyema and psoas muscle abscess (Chang et al., 2005; Maltezou et al., 2009). K. pneumoniae has become a common cause of community acquired bacterial meningitis in adults in Taiwan, in the absence of liver abscess or other fecal infection. Most cases have been reported from Taiwan and studies revealed that K. pneumoniae was the predominant serotype causing liver abscess (Chuang et al., 2006). The entity has also been described in other parts of Asia, North America and Europe (Chuang et al., 2006; Ko et al., 2002; Rahimian et al., 2004; Fang et al., 2005).

These Gram negative bacteria, such as K. pneumonia and Escherichia coli are proved to be the cause of bacterial endophthalmitis in North America and in East Asia. Among these cases, Klebsiella spp is the most common etiologic agent followed by E. coli (Jackson et al., 2003; Wong, 2000), hence becoming the major cause of endogenous endophthalmitis. The resulting inflammation may progress within days and lead to decreased vision, loss of light perception or enucleation despite systemic antibiotics treatment or surgical interventions such as intravitreal antibiotic injections or vitrectomy (Jackson et al., 2003; Yoon et al., 2003). These bacteriae and their components such as lipopolysaccharide (LPS) carry pathogen associated molecular patterns. Recognition of LPS leads to the rapid activation of intracellular signaling pathways, resulting in the release of proinflammatory mediators in mammalian phagocytic cells (Heumann and Roger, 2002). Retinal pigment epithelium (RPE) cells have an important role in different pathologic processes of the retina such as age related macular degeneration, diabetic retinopathy or inherited pathologies (Cai et al., 2000; Lutty et al., 1999; Hamel et. al., 1998; Morimura et al., 1998).

K. pneumoniae, were rarely reported as the monomicrobial cause (Gradon, 1996; Marra and Hotaling, 1996; Parhiscar and Har-El, 2001; Methieu et al., 1995; Sethi and Stanley, 1991). However, several studies of Fascial space infections (FSI) in Taiwan showed that $K$. pneumoniae was not an uncommon pathogen for the disease (Wang et al., 2002; Hsiao and Chao, 1996; Chu et al., 1991; Yuan et al., 1997; Ho and Hsu, 1994; Juang et al., 1989). FSIs of head and neck are of either odontogenic or oropharyngeal origin and extend to potential fascial spaces of the lower part of the head and upper portion of the neck (Margo et al., 1994). Infections of these spaces may intercommunicate with one another and thus potentiate the extension and complication of the disease ( $\mathrm{Lu}$ et al., 1997). Although rare, life threatening complications such as airway obstruction, intracranial or pleuropulmonary extension, and 
haematogenous dissemination or other metastatic foci clearly indicate the potentially serious nature of these infections (Jang et al., 1993).

\section{Medication for $K$. pneumoniae infections}

Infections with Gram negative bacteria are of imminent concern as they are more difficult to treat and visual outcome is poor. K. pneumoniae are major nosocomial pathogens producing extended spectrum betalactamase (ESBL). ESBL producers are usually susceptible only to carbapenems, and these drugs have been the treatment of choice for severe infections by ESBLproducer K. pneumoniae (Paterson and Bonomo, 2005). More recently, the emergence of carbapenemase producing K. pneumoniae (KPC) has severely challenged antimicrobial therapy, since it confers a high level of resistance to all $\beta$-lactams and distinct levels of resistance to carbapenems (Nordmann et al., 2009). As K. pneumoniae is one of the most common nosocomial pathogens, its ability to produce extended spectrum $\beta$-lactamases (ESBLs) has caused great concern worldwide (Falagas et al., 2007; Nordmann et al., 2009). Carbapenem resistance among $K$. pneumoniae, which first emerged a decade ago, continues to spread (Jacoby and Munoz-Price, 2005; MacKenzie et al., 1997; Livermore, 2002; Walsh et al., 2005), and is a cause of major concern.

They can efficiently hydrolyse penicillins, all cephalosporins, monobactams, carbapenems, and even $\beta$-lactamase inhibitors (Papp-Wallace et al., 2010). Many bacteria with these enzymes remain susceptible to colistin, tigecycline and one or more aminoglycoside, but some are resistant even to these drugs. Moreover, only a few drugs are in development against KPCpositive bacteria (Munoz-Price et al., 2013). The ESBL producers can also develop co-resistance to other classes of antimicrobial agents, such as fluoroquinolones, cotrimoxazole and aminoglycosides. Howerver, a recent study showed its inhibition by Fosfomycin, an antibiotic used in the treatment of UTI related infections (Liu et al., 2011).

Epidiomology of the species is discussed (Montgomerie, 1979) and the comparison to its genomics study relating to antibiotics resistance is extensively studied and has revealed its resistance capacity

So, there is an urgent need of introducing new, effective antibiotics with lower side effect, such as natural antibiotic derived from plants. Aggressive treatments and analysis with these natural antibiotics are required to prevent its further spreading.

\section{PLANTS SUPPRESSING THE SURVIVAL OF $K$. pneumoniae}

Nature has been a source of medicinal agents for thousands of years and an impressive number of modern drugs have been isolated from natural sources. This plant based, traditional medicine system continues to play an essential role in health care, with about $80 \%$ of the world's inhabitants relying mainly on traditional medicines for their primary health care (Owolabi et al., 2007). According to World Health Organization, medicinal plants would be the best source to obtain a variety of drugs (Doughari et al., 2008).

Numerous studies demonstrated that medicinal plants are sources of nutrient and non-nutrient compounds, many of which display antioxidant and antimicrobial properties which can protect the human body against both cellular oxidation reactions and pathogens. Thus, it is important to characterize the different types of medicinal plants for their antioxidant and antimicrobial potentials, and such plants should be investigated to better understand their properties, safety and efficacy (Nascimento et al., 2000). The use of medicinal plants as a source for relief from illness can be traced back over five millennia, to written documents of the early civilization in China, India and the north east (Mahesh and Satish, 2008). The potential of higher plants as a source for new drugs is still largely unexplored. Among the estimated 2,50,000- 5,00,000 plant species, only a small percentage have been investigated phytochemically and the fraction submitted to biological or pharmacological screening. Compounds of natural or synthetic origin have been the source of innumerable therapeutic agents (Mahesh and Satish, 2008; Kroschwitz et al., 1992). Medicinal plants are rich sources of antimicrobial agents. A wide range of medicinal plants are used to get different rasayanas which possess different medicinal properties against various microbes (Vashist and Jindal, 2012). Long before mankind discovered the existence of microbes, the idea that certain plants had healing potential, indeed, that they contained what we would currently characterize as antimicrobial principles, was well accepted. Still these traditional medicines are included as part of the habitual treatment of various maladies to treat common infectious diseases (Doughari et al., 2012).

In recent years, research on medicinal plants has attracted a lot of attentions globally. Large body of evidence has accumulated to demonstrate the promising potential of Medicinal Plants used in various traditional, complementary and alternate systems of treatment of human diseases. Plants are rich in a wide variety of secondary metabolites such as tannins terpenoids, alkaloids, flavonoids, etc, which have been found in vitro to have antimicrobial properties (Dahanukar et. al., 2000; Cowan, 1999). Clinical microbiologists have two reasons to be interested in the topic of antimicrobial plant extracts. First, it is very likely that these phytochemicals will find their way into the arsenal of antimicrobial drugs prescribed by the physicians; several are already being tested on humans. Scientists realize that the effective life span of any antibiotic is limited, so new sources, especially plant sources are also being investigated. Second, the public is becoming increasingly aware of the problems with the over prescription and misuse of traditional antibiotics. In addition many people are interested in having more autonomy over their medical care. A multitude of plants compounds (often of unreliable purity) is readily available over the counter from herbal suppliers and national food stores and the self medication with these substances is a common practice to certain extent (Cowan, 1999). In this review we had studied some recent publications to enlist some of the flora which are useful to inhibit the growth of the pathogen, $K$. 
pnuemoniae. 90 plants belonging to 51 families are enlisted in Alphabetical order of the plant name (Table 1). Plant parts such as bark, leaves, roots, fruits were used to extract active components against the organism. Mostly the leaves were used. Solvents such as Methanol, Water, Chloroform, Ethanol, etc., were used to prepare the extracts. The results were compared with standard antibiotics.

\section{PLANT ESSENTIAL OILS AS GROWTH INHIBITORS OF K. pneumoniae}

The spread of drug resistant pathogens is one of the most serious threats to successful treatment of microbial diseases. Down the ages, essential oils and other extracts of plants have evoked interest as sources of natural products. They have been screened for their potential uses as alternative remedies for the treatment of many infectious diseases (Tepe et al., 2004). World Health Organization (WHO) noted that majority of the world's population depends on traditional medicine for primary healthcare. Medicinal and aromatic plants are widely used as medicine and constitute a major source of natural organic compounds.

Plant essential oils and extracts have been used for many thousands of years (Jones, 1996), in food preservation and aromatherapy (Buttner et al., 1996); fragrance industries (Van de Braak and Leijten, 1996); pharmaceuticals, alternative medicine and natural therapies (Reynolds, 1996; Lis-Balchin M, Deans, 1997).

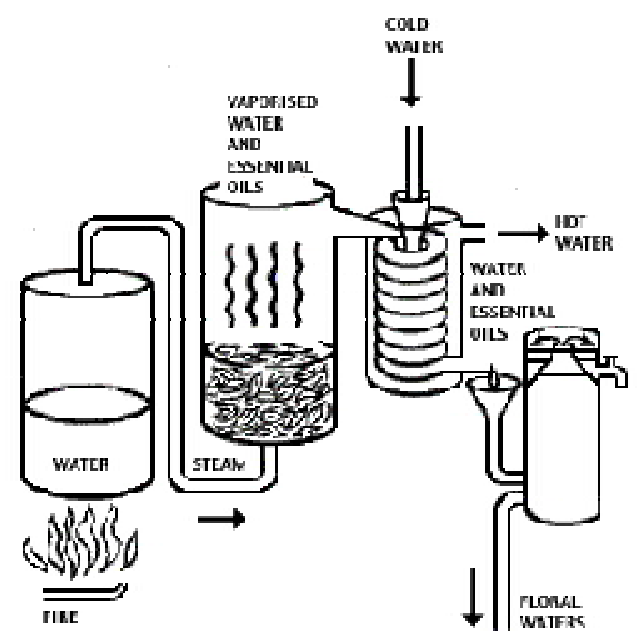

Fig. 2: Extraction of essential oils from plant parts using traditional steam vapor method.

Plants have an almost limitless ability to synthesize aromatic substances. Essential oils (also called volatile oils) are aromatic oily liquids obtained from plant materials (flowers, buds, seeds, leaves, twigs, bark, herbs, wood, fruits and roots) (see traditional essential oil extraction Fig 2). They can be obtained by expression, fermentation or extraction but the method of steam distillation is most commonly used for commercial production. An estimated 3000 different types of essential oils are known, of which 300 are commercially important in fragrance market (Van de Braak and Leijten, 1996). Essential oils are complex mixtures comprising many single compounds. Chemically they are derived from terpenes and their oxygenated compounds. Each of these constituents contributes to the beneficial or adverse effects. Essential oils have been shown to possess antibacterial, antifungal, antiviral insecticidal and antioxidant properties (Burt, 2004; Kordali et al., 2005). Essential oil extracts of various plants have been reported to have inhibitory effects against diverse types of microorganisms including gram-positive bacteria, gram-negative bacteria, fungi and viruses. Many of these plant extracts contain organic chemicals inhibitory for particular microorganisms (Horne et al., 2001).

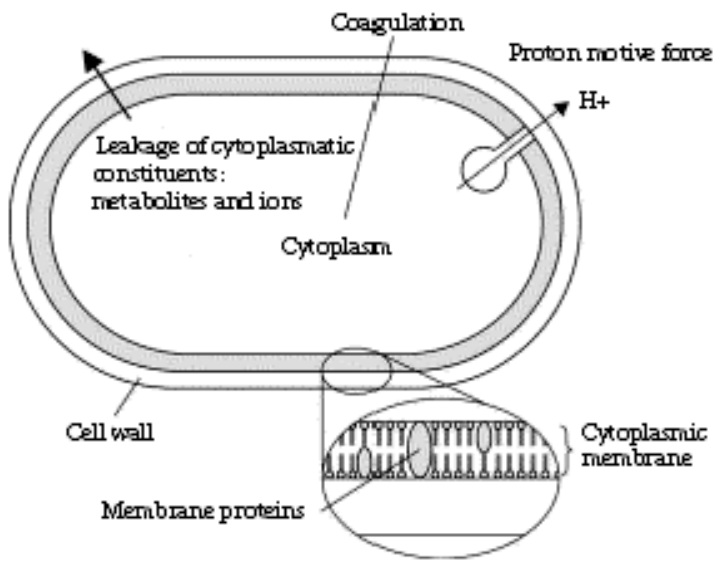

Fig. 3: Sites of action of natural compounds on bacterial cells (Source: Silva and Fernandes Júnior, 2010).

Essential oils such as aniseed, calamus, camphor, cedar-wood, cinnamon, citronella, clove, eucalyptus, geranium, lavender, lemon, lemongrass, lime, mint, nutmeg, orange, palmarosa, rosemary, basil, vetiver and wintergreen have been traditionally used by people for various purposes in different parts of the world. Cinnamon, clove and rosemary oils have shown antibacterial and antifungal activity (Ouattara et. al., 1997); cinnamon oil also possesses antidiabetic property. Anti-inflammatory activity has been found in basil (Singh and Majumdar, 1999). Lemon and rosemary oils possess antioxidant property (Calabrese et al., 1999; Aruoma et al., 1996). Peppermint and orange oils have shown anticancer activity (Kumar et al., 2004; Arias and Ramon-Laca, 2005). Citronella oil has shown inhibitory effect on biodegrading and storage contaminating fungi (De Billerbeck et al., 2001). Lime oil has shown immunomodulatory effect in humans (Arias and Ramon-Laca, 2005). Lavender oil has shown antibacterial and antifungal activity; it was also found to be effective to treat burns and insect bites (Cavanagh and Wilkinson, 2002).

The antimicrobial activity of many essential oils has been previously reviewed and classified as strong, medium or weak (Zaika, 1988). Essential oils are a rich source of biologically active compounds. There has been an increased interest in looking at antimicrobial properties of extracts from aromatic plants particularly essential oils (Milhau et al., 1997). Therefore, it is reasonable to expect a variety of plant compounds in these oils with specific as well as general antimicrobial activity and antibiotic potential (Darokar et al., 1998). An important 
characteristic of essential oils and their components is their hydrophobicity, which enables them to partition the lipids of the bacterial cell membrane and mitochondria, disturbing the cell structures and rendering them more permeable (Knobloch et al., 1986). Extensive leakage from bacterial cells or the exit of critical molecules and ions leads to death (Denyer et al., 1991).
The study revealed different essential oils from plants; 14 essential yielding plants are able to inhibit the growth of the K. pnuemoniae (Table 2; Fig 4). The chemical composition of the essential oils from these plants is enlisted in the Table 3. The structures of essential oil compounds from Allium rotundum, Bupleurum falcatum, etc are shown in the Fig 5.
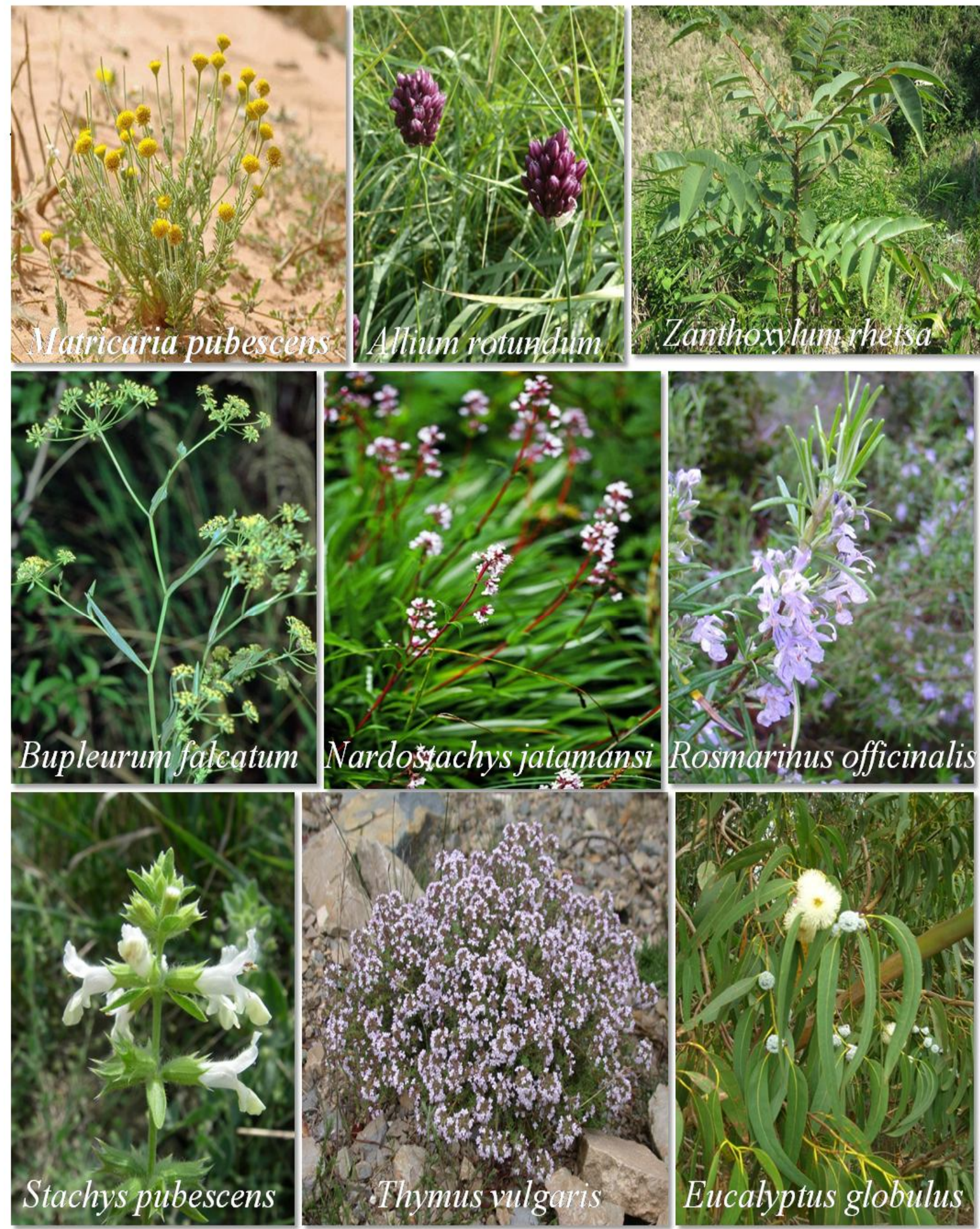

Fig. 4: Essential oil yielding plants showing inhibitory effects on the K. pneumonia. 
<smiles>C=C(C)[C@H]1CC=C(C)CC1</smiles><smiles>O=Cc1ccccc1</smiles><smiles>CC(=O)c1ccccc1</smiles><smiles>CC1=CCC(C(C)C)=CC1</smiles><smiles>Cc1ccc(C(C)C)cc1</smiles>

\section{Limonene Benzaldehyde Acetophenone $\gamma$-Terpinene Cymene}<smiles>C=C1CC/C=C(\C)CC[C@@H]2[C@@H]1CC2(C)C</smiles><smiles>c1ccc2ccccc2c1</smiles>

\section{Caryophyllene Naphthalene}<smiles>C1CC1</smiles>

Cyclopropane<smiles>Cc1ccc(C(C)C)c(O)c1</smiles><smiles>C=CC(=C)CC/C=C(\C)CCC=C(C)C</smiles>

Farnesene<smiles>Cc1cc(C)c(C)c(O)c1C</smiles>

Durenol<smiles>C=CC(C)(O)CCC=C(C)C</smiles>

\section{Linalool}

Fig. 5: Chemical structures of chemical compounds present in plant essential oils showing inhibition of the growth of $K$. pnuemoniae.

Table. 1: Plants with antimicrobial efficacy towards K. pnuemoniae.

\begin{tabular}{|c|c|c|c|c|c|c|c|}
\hline $\begin{array}{l}\text { S. } \\
\text { No. }\end{array}$ & $\begin{array}{l}\text { Plant } \\
\text { name }\end{array}$ & Family & $\begin{array}{c}\text { Plant } \\
\text { part }\end{array}$ & Solvent & Strain & Antibiotic Used & Reference \\
\hline 1. & $\begin{array}{l}\text { Acacia mearnsii De } \\
\text { Wild. }\end{array}$ & Mimosaceae & bark & Methanol & ATCC 10031 & Tetracycline & $\begin{array}{l}\text { Olajuyigbe et al., } \\
2012\end{array}$ \\
\hline 2. & Acacia nilotica $\mathrm{L}$ & Mimosaceae & leaves & Methanol & - & Doxycycline & Mahmood et al., 2012 \\
\hline 3. & Acalypa indica & Euphobiaceae & Leaves & Methanol & MTCC 3384 & Chloramphenicol & Arutselvi et al., 2012 \\
\hline 4. & Achyranthes aspera & Amaranthaceae & Leaves & Methanol & - & Ciprofloxacin & Doss et al., 2012 \\
\hline 5. & Adathoda vasica & Acanthaceae & Leaves & Methanol & MTCC 3384 & Chloramphenicol & Arutselvi et al., 2012 \\
\hline 6. & Adhatoda vasica & Acanthaceae & leaves & Methanol & - & Streptomycin & Singh et al., $2012 \mathrm{a}$ \\
\hline 7. & $\begin{array}{l}\text { Adiantum } \\
\text { capillus-veneris L. }\end{array}$ & Polypodiaceae & $\begin{array}{l}\text { aerial } \\
\text { parts }\end{array}$ & Water and Methanol & - & - & Mahboubi et al., 2012 \\
\hline 8. & Aegle marmelos & Rutaceae & Fruit & $\begin{array}{l}\text { Ethyl acetate, } \\
\text { Methanol }\end{array}$ & ATCC 20063 & - & Maharjan et al., 2012 \\
\hline 9. & Aerva javanica & Amaranthaceae & $\begin{array}{l}\text { Whole } \\
\text { plant }\end{array}$ & $\begin{array}{l}\text { Ethyl Acetate, } \\
\text { Chloroform } \\
\text { Fraction, } \\
\text { Aqueous } \\
\text { Fraction, } \\
\text { Crude } \\
\text { Fraction, } \\
\text { n-Hexane }\end{array}$ & ATCC 700603 & - & Mufti et al., 2012 \\
\hline 10. & Albizia lebbeck $\mathrm{L}$ & Mimosaceae & leaves & Methanol & - & Doxycycline & Mahmood et al., 2012 \\
\hline 11. & Allium сера & Liliaceae & $\begin{array}{l}\text { fresh } \\
\text { bulbs }\end{array}$ & - & $K 24$ & Ciprofloxacin & Stephen et al., 2012 \\
\hline 12. & Allium rotundum & Liliaceae & flower & Methanol & PTCC 1290 & $\begin{array}{l}\text { Valinomycine, } \\
\text { Gentamicine and } \\
\text { Cholramphenicol }\end{array}$ & Dehpour et al., 2012 \\
\hline 13. & Annona squamosa $\mathrm{L}$ & Annonaceae & seeds & $\begin{array}{l}\text { Petroleum ether, } \\
\text { Methanol, } \\
\text { Chloroform }\end{array}$ & - & Streptomycin & Aher et al., 2012 \\
\hline 14. & Argemone mexicana & Papaveraceae & Leaves & Methanol & - & Ciprofloxacin & Doss et al., 2012 \\
\hline 15. & Argemone mexicana & Papaveraceae & leaves & Methanol & - & Streptomycin & Singh et al., $2012 \mathrm{a}$ \\
\hline
\end{tabular}




\begin{tabular}{|c|c|c|c|c|c|c|c|}
\hline 16. & $\begin{array}{l}\text { Asclepias curassavica } \\
\text { Linn. }\end{array}$ & Asclepiadaceae & roots & $\begin{array}{l}\text { Chloroform, } \\
\text { Acetone, Ethanol } \\
\text { and Water }\end{array}$ & MTCC 109 & Streptomycin & Kurdekar et al., 2012 \\
\hline 17. & $\begin{array}{l}\text { Astragalus } \\
\text { atropilosulus } \\
\text { (Hochst.) Bunge } \\
\text { subsp. } \\
\text { Abyssinicus } \\
\text { (Hochst.) J. B. Gillett }\end{array}$ & Fabaceae & leaves & $\begin{array}{l}\text { Acetone, Ethanol, } \\
\text { Methanol, 1/1 } \\
\text { Ethanol/Methanol, } \\
1 / 1 \\
\text { Ethanol/Acetone,1/1 } \\
\text { Acetone/Methanol, } \\
\text { Hot water }\end{array}$ & - & Gentamicin & Sulaiman et al., 2012 \\
\hline 18. & Azadiracta indica & Meliaceae & Leaves & Methanol & MTCC 3384 & Chloramphenicol & Arutselvi et al., 2012 \\
\hline 19. & Barleria lupulina & Acanthaceae & Leaves & Methanol & _- & Ciprofloxacin & Doss et al., 2012 \\
\hline 20. & $\begin{array}{l}\text { Begonia } \\
\text { floccifera Bedd. }\end{array}$ & Begoniaceae & $\begin{array}{l}\text { Fresh } \\
\text { flowers }\end{array}$ & $\begin{array}{l}\text { Phenol, Tannins, } \\
\text { Xanthoproteins, } \\
\text { Steroids, Tannins, } \\
\text { Steroids, } \\
\text { Phytosterols, } \\
\text { Triterpenoids, } \\
\text { Sapogenins, } \\
\text { Coumarins and } \\
\text { Carbohydrates }\end{array}$ & - & Amikacin & $\begin{array}{l}\text { Jeeva and } \\
\text { Antonisamy, } 2012\end{array}$ \\
\hline 21. & Berberis asiatica & Berberidaceae & leaves & Methanol & $\ldots$ & Streptomycin & Singh et al., $2012 \mathrm{a}$ \\
\hline 22. & Brassica oleracea L. & Brassicaceae & & Ethanolic & _ & - & Paul et al., 2012 \\
\hline 23. & $\begin{array}{l}\text { Bridelia retusa } \\
\text { (Linn.) Spreng. }\end{array}$ & Euphobiaceae & Bark & $\begin{array}{l}\text { Chloroform, } \\
\text { Acetone, Ethanol } \\
\text { and Water }\end{array}$ & MTCC 109 & Streptomycin & Kurdekar et al., 2012 \\
\hline 24. & Bupleurum falcatum & Apiaceae & flowers & - & PTCC-1053 & $\begin{array}{l}\text { Ciprofloxacin, } \\
\text { Gentamicin }\end{array}$ & Hadi et al., 2012 \\
\hline 25. & Cannabis sps & Cannabaceae & - & Aqueous & - & $\begin{array}{l}\text { Ceftazidime, } \\
\text { Carbencillin, } \\
\text { Ceftizoxime, } \\
\text { Amikacin, } \\
\text { Cefotaxime, } \\
\text { Ofloxacin, } \\
\text { Gentamicin, } \\
\text { Amoxicillin, } \\
\text { Ciprofloxacin, } \\
\text { Cephalexin, } \\
\text { Chloramphenicol, } \\
\text { Tetracycline }\end{array}$ & Singh et al., 2012b \\
\hline 26. & Capparis spinosa L. & Capparidaceae & flowers & Methanol & ATCC 10031 & - & Mahboubi et al., 2012 \\
\hline 27. & Carica papaya & Caricaceae & seeds & - & KP55; K2; K24 & Ciprofloxacin & Stephen et al., 2012 \\
\hline 28. & Cassia fistula & Caesalpiniaceae & leaves & Methanol & _ & Streptomycin & Singh et al., 2012a \\
\hline 29. & Cenchrus ciliaris & Poaceae & $\begin{array}{l}\text { Whole } \\
\text { Plant }\end{array}$ & Methanol & - & Ciprofloxacin & Doss et al., 2012 \\
\hline 30. & $\begin{array}{l}\text { Cinnamomum } \\
\text { cecidodaphne }\end{array}$ & Lauraceae & Fruits & - & MTCC 4030 & Ciprofloxacin & Bharti et al., 2012 \\
\hline 31. & $\begin{array}{l}\text { Cinnamomum } \\
\text { zeylanicum }\end{array}$ & Lauraceae & leaves & - & PTCC-1053 & $\begin{array}{l}\text { Ciprofloxacin, } \\
\text { Gentamicin }\end{array}$ & Hadi et al.,2012 \\
\hline 32. & Coccinia grandis & Cucurbitaceae & Leaves & Methanol & - & Ciprofloxacin & Doss et al., 2012 \\
\hline 33. & Cola acuminate & Malvaceae & fruits & - & $\begin{array}{l}\text { ATCC 11296; } \\
\text { KP55; } \\
\text { K24 }\end{array}$ & Ciprofloxacin & Stephen et al., 2012 \\
\hline 34. & $\begin{array}{l}\text { Coriandrum sativum } \\
\text { (L.) }\end{array}$ & Apiaceae & fruits & - & - & $\begin{array}{l}\text { Ampicillin, } \\
\text { Tetracycline, } \\
\text { Kannamycin } \\
\end{array}$ & Suganya et al., 2012 \\
\hline 35. & Coriandrum sativum & Apiaceae & Seeds & _- & MTCC 4030 & Ciprofloxacin & Bharti et al., 2012 \\
\hline 36. & Coriandrum sativum & Apiaceae & leaves & - & PTCC-1053 & $\begin{array}{l}\text { Ciprofloxacin, } \\
\text { Gentamicin }\end{array}$ & Hadi et al., 2012 \\
\hline 37. & Carissa opaca & Apocynaceae & leaves & Methanol & - & Streptomycin & Singh et al., $2012 \mathrm{a}$ \\
\hline 38. & $\begin{array}{l}\text { Costus speciosus } \\
\text { (L.) Spreng. }\end{array}$ & Costaceae & Rhizome & $\begin{array}{l}\text { Chloroform, } \\
\text { Acetone, Ethanol } \\
\text { and Water } \\
\end{array}$ & MTCC 109 & Streptomycin & Kurdekar et al., 2012 \\
\hline 39. & Cotinus coggygria & Anacardiacaea & leaves & Methanol & - & Streptomycin & Singh et al., 2012a \\
\hline 40. & $\begin{array}{l}\text { Couroupita } \\
\text { guianensisAubl. }\end{array}$ & Lecythidaceae & Fruits & Chloroform & $\begin{array}{l}\text { MTCC 109; } \\
\text { ESBL } 3971 ; \\
\text { ESBL } 75799 ; \\
\text { ESBL } 3894 ; \\
\text { ESBL } 3967\end{array}$ & Streptomycin & Rauf et al., 2012 \\
\hline 41. & $\begin{array}{l}\text { Cupressus } \\
\text { sempervirens } \mathrm{L} .\end{array}$ & Cupressaceae & $\begin{array}{l}\text { aerial } \\
\text { parts }\end{array}$ & $\begin{array}{l}\text { Methanolic, } \\
\text { Ethanolic and Ethyl } \\
\text { Acetate }\end{array}$ & МТСС 618 & Pencillin & $\begin{array}{l}\text { Chaudhary et al., } \\
2012\end{array}$ \\
\hline
\end{tabular}




\begin{tabular}{|c|c|c|c|c|c|c|c|}
\hline 42. & $\begin{array}{l}\text { Cynometra } \\
\text { travancorica Bedd. }\end{array}$ & Fabaceae & $\begin{array}{l}\text { Whole } \\
\text { plant }\end{array}$ & $\begin{array}{l}\text { Cold water and } \\
\text { Ethanolic }\end{array}$ & - & Ampicilin & John et al., 2012 \\
\hline 43. & Cyperus scariosus & Cyperaceae & Roots & & MTCC 4030 & Ciprofloxacin & Bharti et al., 2012 \\
\hline 44. & $\begin{array}{l}\text { Dactyloctenium } \\
\text { indicum }\end{array}$ & Poaceae & $\begin{array}{l}\text { Whole } \\
\text { Plant }\end{array}$ & Methanol & - & Ciprofloxacin & Doss et al., 2012 \\
\hline 45. & $\begin{array}{l}\text { Datura } \\
\text { stramonium }\end{array}$ & Solanacaea & stem-bark & Ethanolic & KP72011FMC & Gentamycin & Shagal et al., 2012 \\
\hline 46. & $\begin{array}{l}\text { Desmodium } \\
\text { gangeticum }\end{array}$ & Fabaceae & - & $\begin{array}{l}\text { Aqueous, } \\
\text { Methanolic, } \\
\text { Ethanolic }\end{array}$ & - & $\begin{array}{l}\text { Ceftazidime, } \\
\text { Carbencillin, } \\
\text { Ceftizoxime, } \\
\text { Amikacin, } \\
\text { Cefotaxime, } \\
\text { Ofloxacin, } \\
\text { Gentamicin, } \\
\text { Amoxicillin, } \\
\text { Ciprofloxacin, } \\
\text { Cephalexin, } \\
\text { Chloramphenicol, } \\
\text { Tetracycline } \\
\end{array}$ & Singh et al., 2012b \\
\hline 47. & Emblica officinalis & Euphorbiaceae & fruits & $\begin{array}{l}\text { Aqueous, Acetone, } \\
\text { Chloroform, Ethyl } \\
\text { Acetate } \\
\text { and Methanol }\end{array}$ & - & - & Patil et al., 2012 \\
\hline 48. & Eucalyptus globulus & Myrtaceae & Leaves & _ & MTCC 4030 & Ciprofloxacin & Bharti et al., 2012 \\
\hline 49. & Euphorbia hirta & Euphobiaceae & $\begin{array}{l}\text { Leaf, } \\
\text { stem, root } \\
\text { and fruits }\end{array}$ & Alkaloids & MTCC 4030 & - & $\begin{array}{l}\text { Singh and Kumar, } \\
2012\end{array}$ \\
\hline 50. & $\begin{array}{l}\text { Ficus benghalensis } \\
\text { Linn. }\end{array}$ & Moraceae & leaves & $\begin{array}{l}\text { Chloroform, } \\
\text { Methanol }\end{array}$ & MTCC B2405 & Ciproflaxacin & Koon and Rao, 2012 \\
\hline 51. & Ficus sarmentosa & Moraceae & $\begin{array}{l}\text { Whole } \\
\text { plant }\end{array}$ & $\begin{array}{l}\text { n-Hexane, } \\
\text { Chloroform, Ethyl } \\
\text { Acetate and } \\
\text { Methanolic } \\
\end{array}$ & - & Streptomycin & Rauf et al., 2012 \\
\hline 52. & Garcinia kola & Clusiaceae & seeds & - & $\begin{array}{l}\text { ATCC 11296; } \\
\text { KP55; } \\
\text { K24; KP63; K2 }\end{array}$ & Ciprofloxacin & Stephen et al., 2012 \\
\hline 53. & Garcinia lucida & Clusiaceae & seeds & - & $\begin{array}{l}\text { ATCC 11296; } \\
\text { KP55; } \\
\text { K24; KP63; K2 }\end{array}$ & Ciprofloxacin & Stephen et al., 2012 \\
\hline 54. & $\begin{array}{l}\text { Gymnema sylvestre } \\
\text { (Retz) } \\
\text { R. Br ex. Schultes }\end{array}$ & Asclepiadaceae & $\begin{array}{l}\text { Leaf }(\mathrm{L}) \\
\text { and } \\
\operatorname{stem}(\mathrm{S})\end{array}$ & $\begin{array}{l}\text { Petroleum Ether, } \\
\text { Chloroform, } \\
\text { Acetone, Methanol, } \\
\text { Water }\end{array}$ & MTCC 109 & $\begin{array}{l}\text { chloramphenicol and } \\
\text { tetracycline }\end{array}$ & Murugan et al., 2012 \\
\hline 55. & $\begin{array}{l}\text { Hallea ledermannii } \\
\text { (Krause) Verdc. }\end{array}$ & Rubiaceae & leaves & Methanol & - & ciprofloxacin & $\begin{array}{l}\text { Adeleke Adesegun et } \\
\text { al., } 2012\end{array}$ \\
\hline 56. & Isodon rugosus & Lamiaceae & $\begin{array}{l}\text { Whole } \\
\text { plant }\end{array}$ & $\begin{array}{l}\text { n-Hexane, } \\
\text { Chloroform, Ethyl } \\
\text { Acetate and } \\
\text { Methanolic } \\
\end{array}$ & - & Streptomycin & Rauf et al., 2012 \\
\hline 57. & $\begin{array}{l}\text { Lagenandra } \\
\text { Toxicaria Dalz. }\end{array}$ & Araceae & Rhizome & $\begin{array}{l}\text { Chloroform, } \\
\text { Acetone, Ethanol } \\
\text { and Water }\end{array}$ & MTCC 109 & Streptomycin & Kurdekar et al., 2012 \\
\hline 58. & $\begin{array}{l}\text { Lobelia } \\
\text { nicotianaefolia } \\
\text { Roth ex R. \& S } \\
\end{array}$ & Lobeliaceae & $\begin{array}{l}\text { Leaves } \\
\text { and roots }\end{array}$ & $\begin{array}{l}\text { Chloroform, } \\
\text { Acetone, Ethanol } \\
\text { and Water }\end{array}$ & MTCC 109 & Streptomycin & Kurdekar et al., 2012 \\
\hline 59. & $\begin{array}{l}\text { Matricaria pubescens } \\
\text { Desf. }\end{array}$ & Asteraceae & $\begin{array}{l}\text { aerial } \\
\text { parts }\end{array}$ & Aqueous, Ethanolic & CIP 106818 & Ampicillin & Makhloufi et al., 2012 \\
\hline 60. & Medicago sativa & Poaceae & $\begin{array}{l}\text { Whole } \\
\text { Plant }\end{array}$ & Methanol & - & Ciprofloxacin & Doss et al., 2012 \\
\hline 61. & Melaleuca alternifolia & Myrtaceae & Leaves & - & MTCC 4030 & Ciprofloxacin & Bharti et al., 2012 \\
\hline 62. & Melinis repens & Poaceae & $\begin{array}{l}\text { Whole } \\
\text { Plant }\end{array}$ & Methanol & - & Ciprofloxacin & Doss et al., 2012 \\
\hline 63. & $\begin{array}{l}\text { Mimosa himalayana } \\
\text { Gamble }\end{array}$ & Mimosaceae & leaves & Methanol & - & Doxycycline & Mahmood et al., 2012 \\
\hline 64. & $\begin{array}{l}\text { Morinda pubescens } \\
\text { var. pubescens J.E. } \\
\text { Smith }\end{array}$ & Rubiaceae & $\begin{array}{l}\text { Leaf }(\mathrm{L}) \\
\text { and stem } \\
\text { bark (SB) }\end{array}$ & $\begin{array}{l}\text { Petroleum Ether, } \\
\text { Chloroform, } \\
\text { Acetone, Methanol, } \\
\text { Water }\end{array}$ & МТСC 109 & $\begin{array}{l}\text { chloramphenicol and } \\
\text { tetracycline }\end{array}$ & Murugan et al., 2012 \\
\hline 65 & $\begin{array}{l}\text { Murraya paniculata } \\
\text { Linn. }\end{array}$ & Rutaceae & $\begin{array}{l}\text { Whole } \\
\text { plant }\end{array}$ & $\begin{array}{l}\text { Petroleum Ether, } \\
\text { Ethanol, Methanol, } \\
\text { Hydroalcoholic } \\
\text { extracts }\end{array}$ & - & - & Gautam et al., 2012 \\
\hline 66. & Musa paradisiaca & Musaceae & Flower & $\begin{array}{l}\text { Ethanol \& } \\
\text { EtOH:Water }(1: 1)\end{array}$ & - & Amikacin & Jawla et al., 2012 \\
\hline
\end{tabular}




\begin{tabular}{|c|c|c|c|c|c|c|c|}
\hline 72. & $\begin{array}{l}\text { Persicaria piripu } \\
\text { (DC.) M.R.Almeida }\end{array}$ & Polygonaceae & Leaves & $\begin{array}{l}\text { Chloroform, } \\
\text { Acetone, Ethanol } \\
\text { and Water }\end{array}$ & MTCC 109 & Streptomycin & Kurdekar et al., 2012 \\
\hline 73. & Phylanthus niruri & Euphorbiaceae & Leaves & Methanol & MTCC 3384 & Chloramphenicol & Arutselvi et al., 2012 \\
\hline 74. & Picralima nitida & Apocynaceae & fruits & - & $\begin{array}{l}\text { ATCC 11296; } \\
\text { KP55; } \\
\text { K24; KP63; K2 }\end{array}$ & Ciprofloxacin & Stephen et al., 2012 \\
\hline 75. & Polygala grineris & Polygalaceae & Leaves & Methanol & MTCC 3384 & Chloramphenicol & Arutselvi et al., 2012 \\
\hline 76. & Punica granatum L. & Punicaceae & peels & - & - & - & Ullah et al., 2012 \\
\hline 77. & Quercus dilatata & Fagaceae & Fruit & Methanol & $\ldots$ & _- & Sarwat et al., 2012 \\
\hline 78. & $\begin{array}{l}\text { Remusatia vivipara } \\
\text { (Roxb.) Schott \& } \\
\text { Endl. }\end{array}$ & Araceae & Corm & $\begin{array}{l}\text { Chloroform, } \\
\text { Acetone, Ethanol } \\
\text { and Water }\end{array}$ & MTCC 109 & Streptomycin & Kurdekar et al., 2012 \\
\hline 79. & Rhynchosia capitata & Fabaceae & $\begin{array}{l}\text { Whole } \\
\text { Plant }\end{array}$ & Water & - & Ciprofloxacin & Doss et al., 2012 \\
\hline 80. & Ricinus communis & Euphorbiaceae & leaves & Methanol & _ & Streptomycin & Singh et al., $2012 \mathrm{a}$ \\
\hline 81. & $\begin{array}{l}\text { Rosmarinus officinalis } \\
\text { L. }\end{array}$ & Lamiaceae & $\begin{array}{l}\text { aerial } \\
\text { parts }\end{array}$ & - & WHO24 & Ampicillin & Chobba et al., 2012 \\
\hline 82. & $\begin{array}{l}\text { Salvadora oleoides } \\
\text { Decne. }\end{array}$ & Salvadoraceae & stem & Benzene & MTCC 3384 & Streptomycin & Kumar et al., 2012 \\
\hline 83. & Sambucus ebulus L. & Caprifoliaceae & leaves & Water and Methanol & - & - & Mahboubi et al., 2012 \\
\hline 84. & $\begin{array}{l}\text { Sesame } \\
\text { Indicum }\end{array}$ & Pedaliaceae & $\begin{array}{l}\text { White } \\
\text { seeds }\end{array}$ & Aqueous & - & $\begin{array}{l}\text { Ceftazidime, } \\
\text { Carbencillin, } \\
\text { Ceftizoxime, } \\
\text { Amikacin, } \\
\text { Cefotaxime, } \\
\text { Ofloxacin, } \\
\text { Gentamicin, } \\
\text { Amoxicillin, } \\
\text { Ciprofloxacin, } \\
\text { Cephalexin, } \\
\text { Chloramphenicol, } \\
\text { Tetracycline }\end{array}$ & Singh et al., 2012b \\
\hline 85. & Sesame Indicum & Pedaliaceae & $\begin{array}{l}\text { Black } \\
\text { seeds }\end{array}$ & $\begin{array}{l}\text { Aqueous, } \\
\text { Methanolic, } \\
\text { Ethanolic }\end{array}$ & - & $\begin{array}{l}\text { Ceftazidime, } \\
\text { Carbencillin, } \\
\text { Ceftizoxime, } \\
\text { Amikacin, } \\
\text { Cefotaxime, } \\
\text { Ofloxacin, } \\
\text { Gentamicin, } \\
\text { Amoxicillin, } \\
\text { Ciprofloxacin, } \\
\text { Cephalexin, } \\
\text { Chloramphenicol, } \\
\text { Tetracycline }\end{array}$ & Singh et al., 2012b \\
\hline 86. & Solanum nigrum & Solanaceae & Leaves & Methanol & MTCC 3384 & Chloramphenicol & Arutselvi et al., 2012 \\
\hline 87. & Stachys pubescen & Apocynaceae & leaves & - & PTCC-1053 & $\begin{array}{l}\text { Ciprofloxacin, } \\
\text { Gentamicin }\end{array}$ & Hadi et al.,2012 \\
\hline 88. & Tagetes erecta & Asteraceae & leaves & Aqueous & _ & Gentamicin G30 & Dasgupta et al., 2012 \\
\hline 89. & Thymus vulgaris & Labiatae & $\begin{array}{l}\text { Flowerin } \\
\mathrm{g} \text { tips and } \\
\text { Leaves } \\
\end{array}$ & - & MTCC 4030 & Ciprofloxacin & Bharti et al., 2012 \\
\hline 90. & $\begin{array}{l}\text { Trigonella foenum- } \\
\text { graecum }\end{array}$ & Leguminoseae & $\begin{array}{l}\text { Whole } \\
\text { Plant }\end{array}$ & Methanol & - & Ciprofloxacin & Doss et al., 2012 \\
\hline 91. & Withania somnifera & Solanacae & $\begin{array}{l}\text { Ripen } \\
\text { fruits }\end{array}$ & $\begin{array}{l}\text { Glacial Acetic Acid, } \\
\text { Chloroform, } \\
\text { Toluene. }\end{array}$ & MTCC 4030 & Gentamycin & Singariya et al., 2012 \\
\hline 92. & Withania somnifera & solanacae & leaves & $\begin{array}{l}\text { Acetic acid, Ethanol, } \\
\text { Acetone, Ethyl } \\
\text { acetate, Benzene, } \\
\text { Toluene }\end{array}$ & MTCC 4030 & Gentamycin & Singariya et al., 2012 \\
\hline 93. & Withania somnifera & Solanaceae & $\begin{array}{l}\text { Leaf, } \\
\text { stem and } \\
\text { root }\end{array}$ & Alkaloids & MTCC 4030 & - & $\begin{array}{l}\text { Singh and Kumar, } \\
2012\end{array}$ \\
\hline 94. & Woodfordia fruticosa & Lythraceae & Flower & Methanolic & ATCC 20063 & 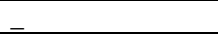 & Maharjan et al., 2012 \\
\hline 95. & Woordfordia fruticosa & Lythraceae & Flower & Methanol & _ & _ & Sarwat et al., 2012 \\
\hline 96. & $\begin{array}{l}\text { Zanthoxylum } \\
\text { armatum }\end{array}$ & Rutaceae & leaves & Methanol & - & Streptomycin & Singh et al., 2012a \\
\hline 97. & Zanthoxylum rhetsa & Rutaceae & Leaves & _ & MTCC 4030 & Ciprofloxacin & Bharti et al., 2012 \\
\hline
\end{tabular}


Table. 2: Essential oil yielding plants showing inhibitory effects towards K. pnиетоniae.

\begin{tabular}{|c|c|c|c|c|}
\hline S. NO. & PLANT NAME & PLANT PART & STRAIN & REFERENCE \\
\hline 1. & Allium rotundum & Flower & PTCC 1290 & Dehpour et al., 2012 \\
\hline 2. & Bupleurum falcatum & Flower & PTCC-1053 & Hadi et al.,2012 \\
\hline 3. & Cinnamomum cecidodaphne & Fruits & MTCC 4030 & Bharti et al., 2012 \\
\hline 4. & Cіппатотит zelanicum & Leaves & PTCC-1053 & Hadi et al.,2012 \\
\hline 5. & Coriandrum sativum & Fruits & - & Suganya et al., 2012 \\
\hline 6. & Coriandrum sativum & Seeds & MTCC 4030 & Bharti et al., 2012 \\
\hline 7. & Coriandrum sativum & Leaves & PTCC-1053 & Hadi et al.,2012 \\
\hline 8. & Cyperus scariosus & Roots & MTCC 4030 & Bharti et al., 2012 \\
\hline 9. & Eucalyptus globulus & Leaves & MTCC 4030 & Bharti et al., 2012 \\
\hline 10. & Matricaria pubescens & Aerial parts & CIP 106818 & Makhloufi et al., 2012 \\
\hline 11. & Melaleuca alternifolia & Leaves & MTCC 4030 & Bharti et al., 2012 \\
\hline 12. & Nardostachys jatamansi & Roots & MTCC 4030 & Bharti et al., 2012 \\
\hline 13. & Rosmarinus officinalis & Aerial parts & WHO24 & Chobba et al., 2012 \\
\hline 14. & Stachys pubescens & Leaves & PTCC-1053 & Hadi et al.,2012 \\
\hline 15. & Thymus vulgaris & Flowering tips and Leaves & MTCC 4030 & Bharti et al., 2012 \\
\hline 16. & Zanthoxylum rhetsa & Leaves & MTCC 4030 & Bharti et al., 2012 \\
\hline
\end{tabular}

Table. 3: Chemical Composition of Essential Oils from plants showing inhibitory effects on the K. pnиетопiae.

\begin{tabular}{|c|c|c|}
\hline $\begin{array}{l}\text { S. } \\
\text { No. }\end{array}$ & $\begin{array}{l}\text { Plant } \\
\text { Name }\end{array}$ & Chemical Composition of Essential Oils \\
\hline 1. & A. rotundum & $\begin{array}{l}\text { Thiophene, 2,3-dimethyl; Thiophene, 2,4-dimethyl; CIS PROPENYL METHYL DISULFIDE; TRANS PROPENYL METHYL } \\
\text { DISULFIDE; Furan, 2-pentyl; Octanal; 1-Hexanol, 2-ethyl; Decane, 3,7-dimethyl; Heptane, 2,3,4-trimethyl; Decane, 3,7-dimethyl; } \\
\text { Nonanal; TRANS-PROPENYL PROPYL DISULFIDE; CIS-PROPENYL PROPYL DISULFIDE; 1-Oxa-4,6-diazacyclooctane-5- } \\
\text { thion; o-DICHLOROBENZENE; DODEMORPH; DEMETON; Decanal; Benzaldehyde, 4-ethyl; Benzene, 1,3-bis(1,1- } \\
\text { dimethylethyl); Tetradecane; PROPAMOCARB; Undecanal; 1,4-Dioxane-2,3-diol; Trisulfide, dipropyl; phthalic } \\
\text { thiothionoanhydride; DEMEPHION; 1-Propene, 3,3'-thiobis; Tetradecane; 5,9-Undecadien-2-one, 6,10-dimethyl; 1-Decene; beta.- } \\
\text { Ionone; Pentacosane; Phenol, 2,4-bis(1,1-dimethylethyl; TERBUTHYLAZINE; Hexadecane; Cyclododecane; Eicosene; Tetradecane; } \\
\text { 2-Pentadecanone, 6,10,14-trimethyl; Dibutyl phthalate; (S)-4,4-Dimethyl-2-(4-methyl-3-cyclohexen-1-yl)-1,5-hexadiene; Phytol; } \\
\text { Octadecane; 14-.BETA.-H-PREGNA; 14-.BETA.-H-PREGNA; 1-Hexacosene; 14-.BETA.-H-PREGNA; Eicosane (CAS); 14- } \\
\text {.BETA.-H-PREGNA; 14-.BETA.-H-PREGNA; 14-.BETA.-H-PREGNA; HAHNFETT; 14-.BETA.-H-PREGNA }\end{array}$ \\
\hline 2. & B. falcatum & $\begin{array}{l}\alpha \text {-Pinene; Pinocarvone; } \alpha \text {-Cubebene; Pinocamphone; Heptanal; cis-Verbenol; Myrtenal; Thyopsene; trans-Pinocarveol; Trans- } \\
\text { Verbenol; Cuparene; Torilenol; Spathulenol; } \alpha \text {-Calacorene; Pentacosane }\end{array}$ \\
\hline 3. & C. zeylanicum & $\begin{array}{l}\text { Benzaldehyde; Phenylmethanal; Benzene Acetaldehyde; Acetophenone; Benzaldehyde dimethyl acetal; Benzeneacetic acid; 2- } \\
\text { Propenal; Cinannamaldehyde Dimethylc Acetale; 2-Propenoic acid; Ortho Metthoxy Cinnamic Aldehyde }\end{array}$ \\
\hline 4. & C. sativum & $\alpha$-Pinene; Benzene; $\gamma$-Terpinene; Cyclopropane; Linalool L; Geranyl acetate \\
\hline 5. & E. globulus & $\begin{array}{l}\alpha \text {-Pinene; Camphene; 1,3,8-p-Menthtriene; Nopinen; } \beta \text {-Myrcene; 3-Carene; Eucalyptol; Fenchyl alcohol; Camphor; Linalool; } \\
\text { Trimethyl benzylalcohol; Terpineol; D- Vebenone; Camphenol; 4- Carene; Berbenone; } \alpha \text {-Bisbolene epoxide; } \delta \text {-3-Carene; } 3 \text {-Carene- } \\
\text { 2-ol; Farnesene }\end{array}$ \\
\hline 6. & M.alternifolia & $\begin{array}{l}\text { Trichloromethane, 1,8-Nonadiyne, } \alpha \text {-Pinene, 1-Cyclooctyne, } \beta \text {-Myrcene, } 2 \text {-Propynylcyclopentane, Cymene, } \alpha \text {-Limonene, } 1,8 \text { - } \\
\text { Cineole, } \alpha \text {-phellandrene, Artemesiatriene, } 6 \text {-Methyl-3-heptyne, Linalool 13.989 12.77, } \alpha \text {-Terpineol, Nitropentane, } 3 \text { - Heptene }\end{array}$ \\
\hline 7. & S. pubescens & $\begin{array}{l}\beta \text {-Pinene; 1,4-Cyclohexadiene; Myrcene; } \alpha \text {-Terpinene; Benzene; Limonene; }(\mathrm{E})-\beta \text {-Ocimene; } \gamma \text {-terpinene; } 3 \text {-Cyclohexen-1-ol; } \\
\text { Linalool; 2,6-Octadien ; Octen-1-ol acetate; } 2,6 \text {-Octadienal; Linalyl acetate; } \delta \text {-Elemene; } \beta \text {-Bourbonene; Naphthalene; } \beta \text {-Gurjunene; } \\
\text { Bicyclogerm acrene; Caryophyllene oxide; Spathulenol; Germacrene }\end{array}$ \\
\hline 8. & T. vulgaris & $\begin{array}{l}\beta \text {-Ketopropane, Trichloro methane- 1,4- hexadiene , 4-Methyl-3- methylene, } 1 \text { - Cyclooctyne, } \beta \text {-Pinene, } \beta \text {-Myrcene, } \beta \text {-Cymene, } \\
\text { Dimethylsuccinate, } \alpha \text {-Terpinene, } 2 \text {-Nonynoic acid, Durenol, Thymol }\end{array}$ \\
\hline
\end{tabular}

\section{CONCLUSION}

In recent years, there has been an increase in Klebsiella infections, especially in hospitals, mostly due to multiple antibiotic resistant strains. It can evade host defenses in normal and impaired hosts and spreads to the upper or lower respiratory tract, provoking correlate infections or it may invade the bloodstream, causing invasive diseases. Besides, it is constantly being studied for the frequency with which resistance occurs against the most common antibiotics. The assays used in the studies are just preliminary assays that were used to evaluate the inhibitory efficacy of the plants and their essential oils. No biological evaluation was performed. Recommended antibiotic for this bacterium are Carbapenemases. But, the antibiotics used to compare the results of were not Carbapenemases. We suggest addition of in-vivo studies using animal models for the biological evaluations and clinical trials before release of such antibiotics would be needed to justify its effect and to further evaluate the potential of these oils as an antibacterial agent in topical or oral applications. The inhibitory effects could be compared to the Carbapenemases or Carbapenemases like antibiotics, to improve the understanding about the action of the antibacterial action of the plant based compoounds.

\section{REFERENCES}

Adeleke Adesegun S, Emmanuel Anyika N, Oluseyi Adekoya T, Godwin Essien S. Antibacterial and antioxidant investigations of Hallea ledermannii leaf extract. Indian J Sci Technol, 2012; 5:1885-1887.

Aher PS, Shinde YS, Chavan PP. In vitro evaluation of antibacterial potential of Annona squamosa 1. against pathogenic bacteria. Int J Pharm Sci., 2012; 3: 1457-1460.

Al-Dhabi NA, Balachandran C, Raj MK, Duraipandiyan V, Muthukumar C, Ignacimuthu S, Khan IA, Rajput VS. Antimicrobial, antimycobacterial and antibiofilm properties of Couroupita guianensis Aubl. Fruit extract. BMC Complement Altern Med, 2012; 12:242. 
Alimirzaee P, Gohari AR, Hajiaghaee R, Mirzaee S, Jamalifar H, Monsef-Esfahani R, Amin GH, Saciduia S, Shahverdi AR. 1-Methyl malate from Berberis integrrimafruits enhances the antibacterial activity of ampicillin against Staphylococcus aureus. Phytother Res, 2009; 23:797800 .

Alrumman SA, Moustafa MFM, Alamri SA. Anti-bacterial and anti-fungal investigation of Astragalus atropilosulus subsp. abyssinicus. Afr J Microbiol Res, 2012; 6:6365-6369.

Arias BA, Ramon-Laca L. Pharmacological properties of citrus and their ancient and medieval uses in the Mediterranean region. J Ethnopharmacol, 2005; 97:89-95.

Aruoma OI, Spencer JP, Rossi R, Aeschbach R, Khan A, Mahmood N, Munoz A, Murcia A, Butler J, Halliwell B. An evaluation of the antioxidant and antiviral action of extracts of rosemary and Provencal herbs. Food Chem Toxicol, 1996; 34:449-456.

Arutselvi R, Balasaravanan T, Ponmurugan P, Suresh P, Ramachandran N. Comparative Studies of Anti-microbial Activity of Turmeric and Selected Medicinal Plant Leaf Extracts Used in Indian Traditional Medicine. J Herbs Spices Med Plants, 2012; 18:231-239.

Bharti P, Bai S, Seasotiya L, Malik A, Dalal S. Antibacterial activity and Chemical Composition of Essential Oils of Ten Aromatic Plants against selected Bacteria. Int J Drug Dev \& Res, 2012; 4:342-351.

Burt SA. Essential oils: their antibacterial properties and potential applications in foods: a review. Inter J Food Microbiol, 2004; 94:223-253.

Buttner MP, Willeke K, Grinshpun SA. 1996. Sampling and analysis of airborne microorganisms. In: Hurst CJ, Knudsen GR, McInerney MJ, Stetzenbach LD, Walter MV, Eds. Manual of Environmental Microbiology. Washington, DC: ASM Press 629-640.

Cai J, Nelson KC, Wu M, Sternberg P Jr, Jones DP. Oxidative damage and protection of the RPE. Prog Retin Eye Res, 2000; 19:205-221.

Calabrese V, Randazzo SD, Catalano C, Rizza V. Biochemical studies on a novel antioxidant from lemon oil and its biotechnological application in cosmetic dermatology. Drugs Exp Clin Res, 1999; 25:219225.

Cavanagh HM, Wilkinson JM. Biological activities of lavender essential oil. Phytother Res, 2002; 16:301-308.

Chang CM, Lu FH, Guo HR, Ko WC. Klebsiella pneumonia fascial space infections of the head and neck in Taiwan: emphasis on diabetic patients and repetitive infections. J Infect, 2005; 50:34-40.

Chaudhary HJ, Shahid W, Bano A, Ullah F, Munis F, Fahad S, Ahmad I. In vitro analysis of Cupressus sempervirens L. plant extracts antibaterial activity. $\mathrm{J}$ med plants res, 2012; 6:273-276.

Chobba IB, Bekir A, Mansour RB, Drira N, Gharsallah N, Kadri A. In vitro Evaluation of Antimicrobial and Cytotoxic Activities of Rosmarinus officinalis L. (Lamiaceae) Essential Oil Cultivated from SouthWest Tunisia. Japs, 2012; 2:034-039.

Chu CC, Hsu CY, Yin NT. Deep neck infections: a retrospective analysis of 85 cases. J Otolaryngol Soc Repub China, 1991; 26:58-68

Chuang YP, Fang CT, Lai SY, Chang SC, Wang JT. Genetic determinants of capsular serotype $\mathrm{K} 1$ of $\mathrm{Kp}$ causing primary pyogenic liver abscess. J Infect Dis, 2006; 193:645-54.

Cowan MM. Plant products as antimicrobial agents. Clin Microbiol Rev, 1999; 12:564-582.

Dahanukar SA, Kulkarni RA, Rege NN. Pharmacology of Medicinal Plants and Natural Products. Indian J Pharmacol, 2000; 32:81118

Darokar MP, Mathur A, Dwivedi S,Bhalla R, Khanuja SPS, Kumar S. Detection of antibacterial activity in the floral petals of some higher plants. Curr Sci,1998; 75:187.

Dasgupta N, Ranjan S, Saha P, Jain R, Malhotra S, Arabi Mohamed Saleh MA. Antibacterial Activity of Leaf Extract of Mexican Marigold (Tagetes erecta ) Against Different Gram Positive and Gram Negative Bacterial Strains. J Pharm Res, 2012; 5:4201-4203.

De Billerbeck VG, Roques CG, Bessiere JM, FonvieilleJL, Dargent R. Effects of Cymbopogon nardus (L.) W. Watson essential oil on the growth and morphogenesis of Aspergillus niger. Can J Microbiol, $2001 ; 47: 9-17$
Debnath S, Dey D, Hazra S, Ghosh S, Ray R, Hazra B. Antibacterial and antifungal activity of Terminalia arjuna Wight \& Arn. bark against ATCC strains and multidrug resistant clinical isolates. J Coastal Life Med, 2013; 1:312-318.

Dehpour A.A., Yousefian M., Jafary Kelarijani S.A., Koshmoo M., Mirzanegad S., Mahdavi V., Mousavi S.E., Shirzad E., Afzali M., Javad Bayani M.J., Olyaei juybari E., Yahyapor M.K. Antibacterial Activity And Composition Of Essential Oils Of FlowerAllium rotundum. Adv Environ Biol, 6(3): 1020-1025, 2012.

Denyer SP, Hugo WB. 1991. Biocide induced damage to the bacterial cytoplasmic membrane. In: Denyer SP, Hugo WB, Eds. Mechanisms of Action of Chemical Biocides. Oxford: The Society for Applied Bacteriology, Technical Series No 27, Oxford Blackwell Scientific Publication 171-188.

Doss A, Muhamed Mubarack H, Vijayasanthi M, Venkataswamy R. In-vitro antibacterial activity of certain wild medicinal plants against bovine mastitis isolated contagious pathogens. Asian J Pharm Clin Res, 2012; 5:90-93.

Doughari JH, El-mahmood AM, Tyoyina I. Antimicrobial activity of leaf extracts of Senna obtusifolia (L). Afr J Pharm Pharmacol, 2008; 2:007-013.

Falagas ME, Rafailidis PI, Kofteridis D, Virtzili S, Chelvatzoglou FC, Papaioannou V, Maraki S, Samonis G, Michalopoulos A. Risk factors of carbapenem resistant Klebsiella pneumoniae infections: a matched case control study. J Antimicrob Chemother, 2007; 60:11241130 .

Fang FC, Sandler N, Libby SJ. Liver abscess caused by magA+ Klebsiella pneumonia in North America. J Clin Microbiol, 2005; 43:991-2.

Gautam MK, Gangwar M, Nath G, Rao CV, Goel RK. In-vitro antibacterial activity on human pathogens and total phenolic, flavonoid contents of Murraya paniculata Linn. Leaves. Asian Pac J Trop Biomed, 2012; S:1660-1663.

Gradon JD. Space occupying and life threatening infections of the head, neck and thorax. Infect Dis Clin North Am, 1996; 10:857-78.

Hadi A, Abolfazl M, Ahmadreza D, Hossein N. Essential oil analysis and Antibacterial activities of some medicinal plants. Int $\mathrm{J}$ Phytomedicine, 2012; 4:212-219.

Hamel CP, Tsilou E, Harris E, Pfeffer BA, Hooks JJ, Detrick B, Redmond TM. A developmentally regulated microsomal protein specific for the pigment epithelium of the vertebrate retina. J Neurosci Res, 1993; $34: 414-425$

Heumann D, Roger T. Initial responses to endotoxins and Gram-negative bacteria. Clin Chim Acta, 2002; 323:59-72.

Ho TC, Hsu CY. Deep neck infection in patients with diabetes mellitus, uremia, or cancer. J Otolaryngol Soc Repub China, 1994; 29: 191-196.

Hooper DC. Emerging mechanisms of fluoroquinolone resistance. Emerg Infect Dis, 2001; 7:337-341.

Horne D, Holm M, Oberg C, Chao S, Gary Young D. Antimicrobial Effects of Essential Oils on Streptococcus pneumoniae. Essent Oil Res, 2001; 13:387-392

Hsiao JR, Chao WY. Space infection of head and neck: an analysis of 80 consecutive cases. J Otolaryngol Soc Repub China, 1996 31:304-10

Jackson TL, Eykyn SJ, Graham EM, Stanford MR. Endogenous bacterial endophthalmitis: a 17-year prospective series and review of 267 reported cases. Surv Ophthalmol, 2003; 48:403-423.

Jackson TL, Eykyn SJ, Graham EM, Stanford MR. Endogenous bacterial endophthalmitis: a 17-year prospective series and review of 267 reported cases. Surv Ophthalmol, 2003;48:403-423.

Jacoby GA, Munoz-Price LS. The new beta-lactamases. N Engl J Med, 2005; 352:380-391.

Jang TN, Wang FD, Wang LS, Yu KW, Liu CY. Gram-negative bacillary meningitis in adults: a recent six-year experience. J Formos Med Assoc, 1993; 92:540-6.

Jawla S, Kumar Y, Khan MSY. Antimicrobial and antihyperglycemic activities of Musa paradisiaca flowers. Asian Pac J Trop Biomed, 2012; S:914-918. 
Jeeva S, Marimuthu Antonisamy J. Anti-bacterial and phytochemical studies on methanolic extracts of Begonia floccifera Bedd. Flower. Asian Pac J Trop Biomed, 2012; S:151-154.

John J, Ragi PR, Sujana KA, Anil Kumar N. Analysis of Phytochemical Contents and Antibacterial Activity of an Endangered Tree (Cynometra travancorica Bedd.) of Western Ghats, India. Adv in Biol Res, 2012; 6:01-05.

Jones FA. Herbs-useful plants. Their role in history and today. Euro J Gastroenterol Hepatol, 1996, 8:1227-1231.

Juang YC, Cheng DL, Wang LS, Liu CY, Duh RW, Chang CS. Ludwig's angina: an analysis of 14 cases. Scand J Infect Dis, 1989; $21: 121-5$

Keynan Y, Rubinstein E. The changing face of Klebsiella pneumoniae infections in the community. Int J Antimicrob Ag, 2007; 30:385-389.

Knobloch K, Weigand H, Weis N, Schwarm HM, Vigenschow H. 1986. Action of terpenoids on energy metabolism. In: Brunke EJ De Gruyter, ed. Progress in Essential Oil Research. Berlin: 16th International Symposium on Essential Oils 429-445.

Ko WC, Paterson DL, Sagnimeni AJ, Hansen DS, Gottberg AV, Mohapatra S, Casellas, Goossens H, Mulazimoglu L, Trenholme G, Klugman KP, McCormack JG, Yu VL. Community acquired Klebsiella pneumoniae bacteremia: global differences in clinical patterns. Emerg Infect Dis, 2002; 8:160-6.

Koon SJ, Rao BS. In vitro evaluation of antibacterial activity of crude extracts of Ficus benghalensis Linn., the banyan tree leaves. Indian J natural products resources, 2012; 3:281-284.

Kordali S, Kotan R, Mavi A, Cakir A, Ala A, Yildirim A. Determination of the chemical composition and antioxidant activity of the essential oil of Artemisia dracunculus and of the antifungal and antibacterial activities of Turkish Artemisia absinthium, A. racunculus, Artemisia santonicum, and Artemisia spicigera essential oils. J Agric Food Chem, 2005; 53:9452-9458.

Kroschwitz JI, Howetgrant M, Kirkothmer. Encyclopedia of Chem technol, 1992; 2:893

Kumar A, Samarth RM, Yasmeen S, Sharma A, Sugahara T, Terado T, Kimura H. Anticancer and radioprotective potentials of Mentha piperita. Biofactors, 2004; 22:87-91.

Kumar S, Dhankhar S, Arya VP, Yadav S, Yadav JP. Antimicrobial activity of Salvadora oleoides Decne. against some microorganisms. J Med Plants Res, 2012; 6:2754-2760.

Kurdekar RR, Hedge GR, Hebbar SS. Antimicrobial efficacy of Bridelia retusa (Linn.) Spreng. And Asclepias curassavica Linn. Indian J natural products resources, 2012; 3:598-593.

Kurdekar RR, Hegde GR, Hegde G,. Hebbar SS. Antimicrobial screening of Medicinal plants against human Pathogens- A Comparative account of two different methods of Extraction. Int J Drug Dev \& Res, 2012; 4: 82-89.

Lacmata ST, Kuete V, Dzoyem JP, Tankeo SB, GeraldNgo Teke, Kuiate JR, Pages JM. Antibacterial Activities of Selected Cameroonian Plants and Their Synergistic Effects with Antibiotics against Bacteria Expressing MDR Phenotypes. Evidence Based Complement Altern Med, 2012; 1-11.

Lin RD, Chin YP, Lee MH. Antimicrobial activities in combination with natural flavonoids against clinical extended spectrum b-lactamase producing Klebsiella pneumoniae. Phytother Res, 2005;19: 612-617.

Lis-Balchin M, Deans SG. Bioactivity of selected plant essential oils against Listeria monocytogenes. J Appl Bacteriol, 1997; 82:759-762.

Liu HY, Lin HC, Lin YC, Yu SH, Wu WH, Lee YJ. Antimicrobial susceptibilities of urinary extended-spectrum betalactamase producing Escherichia coli andKlebsiella pneumonia to fosfomycin and nitrofurantoin in a teaching hospital in Taiwan. $\mathrm{J}$ Microbiol, Immunol Infect, 2011; 44:364-368.

Livermore DM. The impact of carbapenemases on antimicrobial development and therapy. Curr Opin Invest Drugs, 2002; 3:218-224.

$\mathrm{Lu} \mathrm{CH}$, Chang WN, Wu HS. Klebsiella pneumonia meningitis: analysis on clinical features of thirty-two adult patients. Chin Med J, 1997; 60:296-302.
Lutty G, Grunwald J, Majji AB, Uyama M, Yoneya S. Changes in choriocapillaris and retinal pigment epithelium in age related macular degeneration. Mol Vis, 1999; 5:35.

MacKenzie FM, Forbes KJ, Dorai-John T, Amyes SJ, Gould IM. Emergence of a carbapenem resistant Klebsiella pneumoniae. Lancet, 1997; 350:783.

Maharjan N, Singh A, Manandhar MD, Basnyat S, Lekhak B, Kalauni SK. Evaluation of Antibacterial Activities of Medicinal Plants. Nepal J Sci Technol, 2012; 13:209-214.

Mahboubi A, Kamalinejad M, Shalviri M, Karbasi Z, Jafariazar Z, Asgharian R. Evaluation of antibacterial activity of three Iranian medicinal plants. African J Microbiol Res, 2012; 6:2048-2052.

Mahesh B, Satish S. Antimicrobial Activity of Some Important Medicinal Plant Against Plant and Human Pathogens. World J Agricultural Sci, 2008; 4:839-843.

Mahmood A, Mahmood A, Qureshi RA. Antimicrobial activities of three species of family mimosaceae. Pak J Pharm Sci, 2012; 25:203-206.

Makhloufi A, Moussaoui A, Lazouni HA. Antibacterial activities of essential oil and crude extracts from Matricaria pubescens (Desf.) growing wild in Bechar, South west of Algeria. J Med Plants Res, 2012; 6:3124-3128.

Maltezou HC, Giakkoupi P, Maragos A, Bolikas M, Raftopoulos V, Papahatzaki H, Vrouhos G, Liakou V, Vatopoulos AC. Outbreak of infections due to KPC-2-producing Klebsiella pneumonia in a hospital in Crete (Greece). J Infect, 2009; 58:213-21.

Margo CE, Mames RN, Guy JR. Endogenous Klebsiella pneumoniae endophthalmitis: report of two cases and review of the literature. Ophthalmol, 1994; 101:1298-301.

Marra S, Hotaling AJ. Deep neck infections. Am J Otolaryngol, 1996; 17:287-98.

Methieu D, Neviere R, Teillon C, Chagnon JL, Lebleu N, Wattel F. Cervical necrotizing fasciitis: clinical manifestations and management. Clin Infect Dis, 1995; 21:51-6.

Milhau G, Valentin A, Benoit F, Mallie M, Bastide J, Pelissier $\mathrm{Y}$, Bessiere J. In vitro antimicrobial activity of eight essential oils. J Essent Oil Res, 1997; 9:329-333.

Montgomerie JZ. Epidemiology of Klebsiella and Hospital Associated Infections. Revs Infectious Diseases, 1979; 1:736-753.

Morimura H, Fishman GA, Grover SA, Fulton AB, Berson EL, Dryja TP. Mutations in the RPE65 gene in patients with autosomal recessive retinitis pigmentosa or leber congenital amaurosis. Proc Natl Acad Sci, 1998; 95:3088-3093.

Mufti FUD, Ullah H, Bangash A, Khan N, Hussain S, Ullah F, Jamil M, Jabeen M. Antimicrobial activities of Aerva javanica and Paeonia emodi plants. Pak J Pharm Sci, 2012; 25:565-569.

Munoz-Price LS, Poirel L, Bonomo RA, Schwaber MJ, Daikos GL, Cormican M, Cornaglia G, Garau J, Gniadkowski M, Hayden MK, Kumarasamy K, Livermore DM, Maya JJ, Nordmann P, Patel JB, Paterson DL, Pitout J, Villegas MV, Wang H, Woodford N, Quinn JP. Clinical epidemiology of the global expansion of Klebsiella pneumoniae carbapenemases. Lancet Infect Dis, 2013; 13:785-96.

Murugan M, Mohan VR, Thamodharan V. Phytochemical screening and antibacterial activity of Gymnema sylvestre (Retz) $\mathrm{R}$. Br ex. Schultes and Morinda pubescens J.E. Smith var. pubescens. J App Pharm Sci, 2012; 2:73-76.

Nascimento GGF, Lacatelli J, Freitas PC, Silva GL. Antibacterial activity of plant extracts and phytochemicals on antibiotic resistant bacteria. Braz J Microbiol, 2000; 31: 886-891.

Nordmann P, Cuzon G, Naas T. The real threat of Klebsiella pneumoniae carbapenemase-producing bacteria. Lancet Infect Dis, 2009; 9:228-236.

Nordmann P, Cuzon G, Naas T. The real threat of Klebsiella pneumoniae carbapenemase-producing bacteria. Lancet Infect Dis, 2009; 9:228-36.

Olajuyigbe OO, Afolayan AJ. In Vitro Antibacterial and TimeKill Assessment of Crude Methanolic Stem Bark Extract of Acacia mearnsii De Wild against Bacteria in Shigellosis. Molecules, 2012; 17:2103-2118 
Oliveira LMde, Nayra da Silva Negreiros Cardoso, Fernandez LG, Milton Ricardo de Abreu Roque, José Raniere Ferreira de Santana, Claudinéia Regina Pelacani and Renato Delmondez de Castro. The effect of growing conditions on phenolic compounds and antimicrobial activity of Myracrodruon urundeuva Fr. Allemão. African J Biotech, 2012; 11:9770-9775.

Ouattara B, Simard RE, Holley RA, Pitte GJP, Begin A. Antibacterial activity of selected fatty acids and essential oils against six meat spoilage organisms. Inter J Food Microbiol, 1997; 37:155-162.

Owolabi J, Omogbai EKI, Obasuyi O. Antifungal and antibacterial activities of the ethanolic and aqueous extract of Kigelia africana (Bignoniaceae) stem bark. Afr J Biotechnol, 2007; 6:882-85.

Papp-Wallace KM, Bethel CR, Distler AM, Kasuboski C, Taracila M, Bonomo RA. Inhibitor resistance in the KPC-2 betalactamase, a preeminent property of this class A beta-lactamase. Antimicrob Agents Chemother, 2010; 54:890-97.

Parhiscar A, Har-El G. Deep neck abscess: a retrospective of 210 cases. Ann Otol Rhinol Laryngol, 2001;110:1051-4.

Paterson DL, Bonomo RA. Extended spectrum beta-lactamases: a clinical update. Clin Microbiol Rev, 2005;18:657-86.

Patil SG, Deshmukh AA, Padol AR, Kale DB. In vitro antibacterial activity of Emblica officinalis fruit extract by tube Dilution Method. Int J Toxicol App Pharmacol, 2012; 2:49-51.

Paul S, Emran TB, Saha D, Zahid Hosen SM. Phytochemical and antimicrobial activity of the plant extracts of Brassica oleracea against selected microbes. Asian J Pharmacy Medical Sci, 2012; 2:40-44.

Rahimian J, Wilson T, Oram V, Holzman RS. Pyogenic liver abscess: recent trends in etiology and mortality. Clin Infect Dis, 2004; $1: 1654-9$.

Rauf A, Muhammad N, Khan A, Uddin N, Atif M, Barkatullah. Antibacterial and Phytotoxic Profile of Selected Pakistani Medicinal Plants. World App Sci J, 2012; 20:540-544.

Reynolds JEF. 1996. Martindale - the Extra Pharmacopoeia. London: Royal Pharmaceutical Society of Great Britain.

Sarwat, Shinwari ZK, Ahmad N. Screening of potential medicinal plants from district sawat specific for controlling women diseases. Pak J Bot, 2012; 44:1193-1198.

Sethi DS, Stanley RE. Parapharygeal abscess. J Laryngol Otol, 1991; 105:1025-30.

Shagal MH, Modibbo UU, Liman AB. Pharmacological justification for the ethnomedical use of Datura Stramonium stem-bark extract in treatment of diseases caused by some pathogenic bacteria. International Research of Pharmacy and Pharmacology, 2012; 2:016-019.

Shahverdi AR, Rafii F, Tavassoli F, Bagheri M, Attar F, Ghahraman A. Piperitone from Mentha longifolia var. chorodictya Rech F. reduces the nitrofuranto in resistance of strains of enterobacteriaceae. Phytother Res, 2004; 18:911-914.

Si H, Hu S, Lin Z, Zeng ZL. Antimicrobial effect of oregano essential oil along and in combination with antibiotics against extended spectrum b-lactamase-producing Escherichia coli. FEMS Immunol Med Microbiol, 2008; 53:190-194.

Sikkema J, De Bont JAM, Poolman B. Interactions of cyclic hydrocarbons with biological membranes. J Biol Chem, 1994; 269:80228028 .

Silva NCC, Fernandes Júnior A. Biological properties of medicinal plants: a review of their antimicrobial activity. J Venom Anim Toxins incl Trop Dis, 2010; 16:402-413.

Singariya P, Kumar P, Krishan Kumar Mourya KK. Evolution of Antimicrobial Activity of Leaf extracts of Winter Cheery (Withania somnifera). Int J PharmTech Res, 2012; 4:1247-1253.

Singariya P, Kumar P, Mourya KK. Ripen fruits of indian ginseng: phyto-chemical and pharmacological examination against human and plant pathogens. International Journal of Applied Biology and Pharmaceutical Technology, 2012; 3:1-8.
Singh G, Kumar P. antibacterial potential of alkaloids of Withania somnifera L. \& Euphorbia hirta L. Int J Pharm Pharm Sci, 2012; 4:78-81.

Singh S, Majumdar DK. Effect of Ocimum sanctum fixed oil on vascular permeability and leucocytes migration. Indian J Exp Biol, 1999; 37:1136-1138.

Singh SK, Vishnoi R, Dhingra GK, Kishor K. Antibacterial activity of leaf extracts of some selected traditional medicinal plants of Uttarakhand, North East India. J Appl Natural Sci, 2012a; 4:47-50.

Singh V, Thakur K, CHAUHAN PK. Effect of poly herbal formulation against klebsiella pneumonia causing pneumonia in children's. Asian J Pharm Clin Res, 2012b; 5:69-75.

Suganya S, Bharathidasan R, Senthilkumar G, Madhanraj P, Panneerselvam A. Antibacterial activity of essential oil extracted from Coriandrum sativam(L.) and GC-MS analysis. J Chem Pharma Res, 2012; 4:1846-1850.

Tepe B, Daferera D, Sokmen M, Polissiou M, Sokmen A. In vitro antimicrobial and antioxidant activities of the essential oils and various extracts of Thymus eigii M. Zohary et P.H. Davis. J Agric Food Chem, 2004; 52:1132-1137.

Ullah N, Ali J, Khan FA, Khurram M, Hussain A, Inayat-urRahman, Zia-ur-Rahman, Shafqatullah. Proximate Composition, Minerals Content, Antibacterial and antifungal Activity Evaluation of Pomegranate (Punica granatum L.) Peels Powder. Middle East J Sci Res, 2012; 11:396401.

Van de Braak SAAJ, Leijten GCJJ. Essential Oils and Oleoresins: A Survey in the Netherlands and other Major Markets in the European Union. CBI, Centre for the Promotion of Imports from Developing Countries, Rotterdam, 1999; 116.

Vashist H, Jindal A. Antimicrobial Activities of Medicinal Plants -Review. Int J Res Pharma Biomed Sci, 2012; 3:222-230.

Walsh TR, Toleman MA, Poirel L, Nordmann P. Metallo-betalactamases: the quiet before the storm?. Clin Microbiol Rev, 2005; 18:306325.

Wang LF, Kuo WR, Lin CS, Lee KW, Huang KJ. Space infections of the head and neck. Kaohsiung J Med Sci, 2002; 18:386-92.

Wong JS, Chan TK, Lee HM, Chee SP. Endogenous bacterial endophthalmitis: an east Asian experience and a reappraisal of a severe ocular affliction. Ophthalmology, 2000; 107:1483-1491.

Wu HS, Wang FD, Tseng CP, Wu TH, Lin YT, Fung CP. Characteristics of healthcare associated and community acquired Klebsiella pneumoniae bacteremia in Taiwan. J Infect, 2012; 64:162-168.

Yala D, Merad AS, Mohamedi D, Ouar Korich MN. Classification et mode d'action des antibiotiques. Médecine du Maghreb, 2001; 91:5-12.

Yoon YH, Lee SU, Sohn JH, Lee SE. Result of early vitrectomy for endogenous Klebsiella neumoniae endophthalmitis. Retina, 2003, 23:366-370

Yuan BC, Lin CZ, Chang P. Deep neck infections. J Otolaryngol Soc Repub China, 1987; 22:73-79.

Zaika LL. Spices and herbs: their antibacterial activity and its determination. J Food Saf, 1988; 23:97-118.

Ziegler J. 2005. Fayard, ed. L'Empire de la honte. France, Paris.

\section{How to cite this article:}

Gowsiya Shaik, Sujatha N and Santosh Kumar Mehar. Medicinal Plants as Source of Antibacterial Agents to Counter Klebsiella pneumoniae. J App Pharm Sci, 2014; 4 (01): 135-147. 